\title{
Cronología de la ocupación humana en los canales septentrionales de Patagonia occidental, Chile
}

\author{
Omar Reyes, César Méndez y Manuel San Román \\ Recibido 14 de marzo 2019. Aceptado 25 de junio 2019
}

\begin{abstract}
RESUMEN
Un programa sistemático de fechados radiocarbónicos en el archipiélago de los Chonos, en los canales noroccidentales de Patagonia, nos permite conocer mejor la ocupación de este territorio por parte de cazadores recolectores marinos. Evaluamos la distribución temporal de estas ocupaciones y la dinámica del poblamiento utilizando la información cronológica para comprender la actividad humana en el paisaje. Las muestras se obtuvieron de una variedad de sitios arqueológicos que abarcan los últimos 6200 años. Estas incluyen estratos enterrados debajo de suelos orgánicos, refugios rocosos, entierros individuales y osarios, y depósitos de concheros a cielo abierto, algunos de los cuales fueron formados por la recolección reciente de moluscos. Discutimos tanto la primera evidencia de la ocupación de este territorio como la continuidad o discontinuidad de su uso a lo largo del tiempo. Evaluamos la intensidad de la señal cronológica humana desde el Holoceno medio hasta momentos recientes y discutimos las posibles explicaciones para las (dis)continuidades en el uso del espacio archipelágico.
\end{abstract}

Palabras clave: Cazadores recolectores marinos; Cronología radiocarbónica; Patagonia occidental; Holoceno medio y tardío.

\begin{abstract}
CHRONOLOGY OF THE HUMAN OCCUPATION OF THE NORTHERN WATER COURSES OF WESTERN PATAGONIAN, CHILE. A systematic radiocarbon dating program in the Chonos Archipelago in the northwestern channels of Patagonia has enabled a better understanding of the occupation of this area by marine hunter-gatherers. The temporal distribution and the population dynamics of these occupations are evaluated by using chronological information to understand human activity across the landscape. Samples were obtained from a variety of archaeological sites spanning the last 6200 years. These include dates from strata buried beneath organic soils, rock shelters, individual burials and ossuaries, and open-air shell middens, some of which were formed by recent shellfish harvesting. Both the evidence of the initial occupation of this territory, as well as the continuity or discontinuity in its use over time, are discussed. The intensity of the human chronological signature starting during the Mid-Holocene and continuing until recent times is evaluated and possible explanations for the (dis)continuities in the use of the archipelagic space are discussed.
\end{abstract}

Keywords: Maritime hunter-gatherers; Radiocarbon chronology; Western Patagonian Channels; Mid and Late Holocene.

Omar Reyes. Centro de Estudios del Hombre Austral, Instituto de la Patagonia, Universidad de Magallanes. Bulnes 0I890, Punta Arenas, Chile. E-mail: omarreyesbaez@gmail.com

César Méndez. Centro de Investigación en Ecosistemas de la Patagonia. Moraleda I6, Coyhaique, Chile. E-mail: cesar.mendez@ciep.cl Manuel San Román. Centro de Estudios del Hombre Austral, Instituto de la Patagonia, Universidad de Magallanes. Bulnes 01890, Punta Arenas, Chile. E-mail:msanromanbontes@gmail.com 


\section{INTRODUCCIÓN}

Las investigaciones arqueológicas realizadas en los canales septentrionales de Patagonia, particularmente en el archipiélago de los Chonos ( 43-46 ${ }^{\circ}$ S, Aisén, Chile), han permitido incorporar extensas áreas insulares a la discusión sobre la diversidad de la adaptación marina por parte de grupos cazadores recolectores pescadores. Las características y diversidad de sitios arqueológicos registrados, junto con sus conjuntos tecnológicos (Porter 1993; Reyes et al. 2015), sus emplazamientos en diversas geoformas marinas (Reyes et al. 2016, 2018), además de los resultados isotópicos sobre restos faunísticos y humanos (Reyes 2017; Reyes et al. 2019), son testimonio de la especialización marina y costera por parte de los grupos canoeros que ocuparon dicho territorio.

Este archipiélago corresponde a un extenso sistema insular con un paisaje discontinuo moldeado por la actividad tectónica, volcánica, glaciar y por los cambios globales de los niveles marinos (Reyes et al. 2018) (Figura 1). Las distancias marinas de entre 40 y $20 \mathrm{~km}$ que lo separan del borde continental, junto con el macizo andino, bosques montanos y volcanes limitan los espacios habitables y el contacto por vía terrestre con otros grupos culturales (Reyes et al. 2015). Comparaciones de datos arqueológicos, bioantropológicos y moleculares apoyan la hipótesis que considera a la costa e interior como dos áreas separadas desde el principio de sus secuencias de ocupación (Méndez y Reyes 2015; Reyes et al. 2015; De la Fuente et al. 2018; Kuzminsky et al. 2018; Méndez et al. 2018). Es por ello que, para la comprensión de las dinámicas ocupacionales en una región tan fragmentada, se precisa de un análisis de la temporalidad de estos procesos fundado en la incorporación de datos cronométricos de la mayor cantidad de zonas posibles. En este trabajo nos proponemos evaluar la desigual distribución de la señal arqueológica humana a partir de la consideración de la distribución de fechados radiocarbónicos. Estos pueden ser considerados como artefactos autodatados que permiten evaluar la magnitud de la presencia humana, en tanto la producción de material fechable puede ser comprendida como dependiente de la mayor o menor actividad de esta (Rick 1987).

Sostenemos que la distribución del registro arqueológico del archipiélago obedecería tanto a

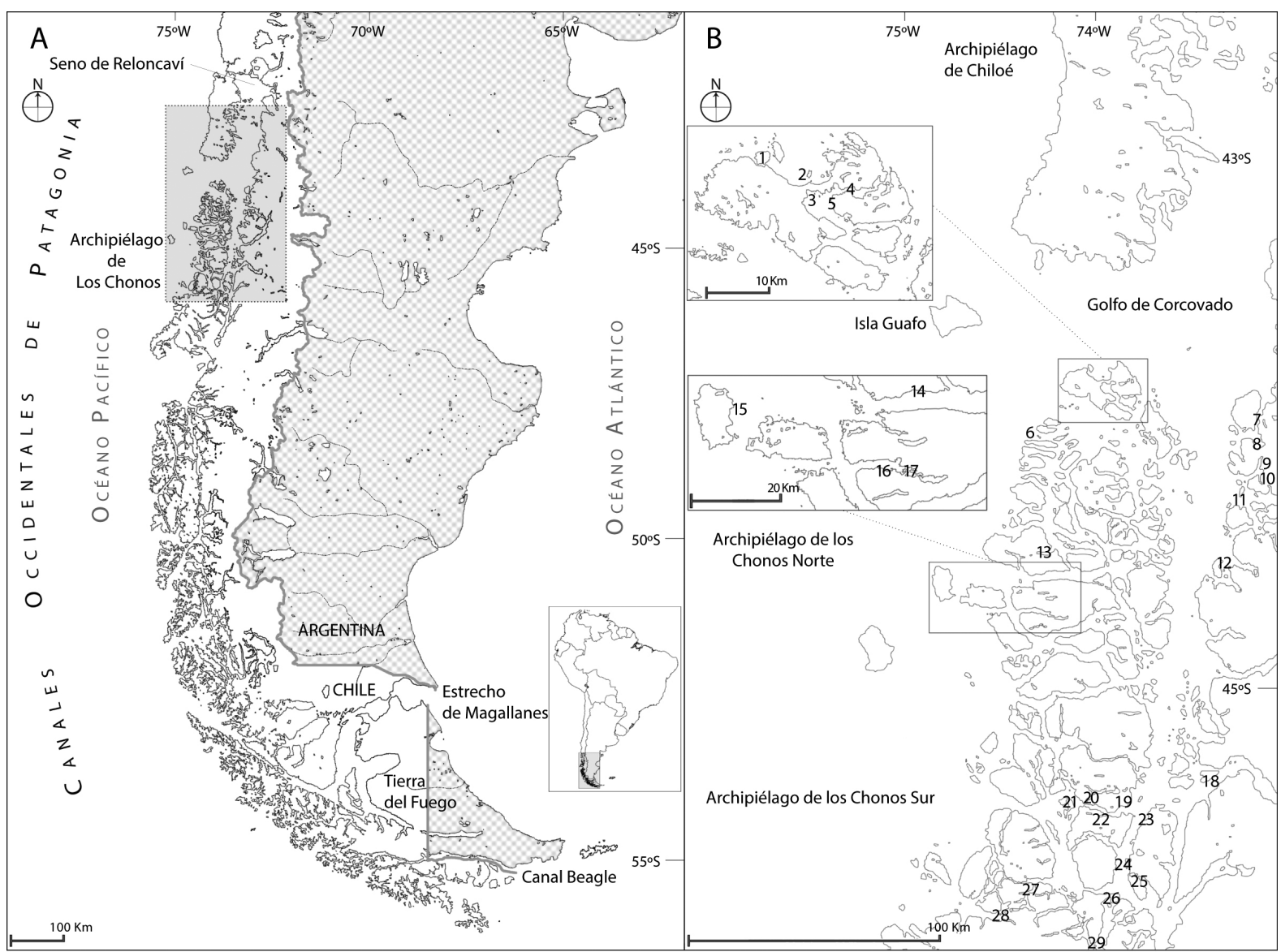

Figura 1. Mapa del área de estudio. A. Patagonia occidental. B. Archipiélago de los Chonos y sitios mencionados en el texto y en Tabla 1. 
Cronología de la ocupación humana en los canales septentrionales de Patagonia occidental... Intersecciones en Antropología 20 (2), agosto-diciembre. 2019. ISSN-e 1850-373X

\begin{tabular}{|c|c|c|c|c|c|}
\hline Sitios & Cód. Laboratorio & Edad & $\delta$ C (\%o) & $2 \sigma$ cal. AP & Material \\
\hline \multirow{2}{*}{ (1) Isla Marta 1} & UGAMS 26290 & $1520 \pm 25 \mathrm{AP}$ & $-22,3$ & $1310-1406$ & Colágeno \\
\hline & D-AMS 006068 & $2074 \pm 30$ AP & 3,3 & 1293-1515 & Valva \\
\hline \multirow{5}{*}{ (2) Alero Low } & UGAMS 26283 & $820 \pm 25 \mathrm{AP}$ & $-11,4$ & $285-453$ & Colágeno \\
\hline & UGAMS 26284 & $990 \pm 25 \mathrm{AP}$ & $-11,2$ & $442-561$ & Colágeno \\
\hline & D-AMS $017799\left(^{*}\right)$ & $1671 \pm 28 \mathrm{AP}$ & $(-)$ & $1430-1583$ & Carbón \\
\hline & UGAMS 26285 & $1940 \pm 25 \mathrm{AP}$ & $-22,6$ & 1747-1906 & Colágeno \\
\hline & UGAMS 26286 & $2050 \pm 25 \mathrm{AP}$ & $-19,4$ & $1901-2012$ & Colágeno \\
\hline \multirow{2}{*}{$\begin{array}{l}\text { (3) Gran Guaiteca } 2 \\
\text { Conchal }\end{array}$} & UGAMS 8294 & $830 \pm 30 \mathrm{AP}$ & $-13,9$ & $287-463$ & Colágeno \\
\hline & D-AMS 006069 & $1963 \pm 25 \mathrm{AP}$ & $-6,1$ & 1198-1389 & Valva \\
\hline (3) Gran Guaiteca 2 Terraza & D-AMS 006073 & $3958 \pm 24 \mathrm{AP}$ & $-22,8$ & $4243-4428$ & Carbón \\
\hline (3) Gran Guaiteca 3 & D-AMS 006070 & $1929 \pm 28 \mathrm{AP}$ & $-0,6$ & $1165-1359$ & Valva \\
\hline (3) Gran Guaiteca 4 & D-AMS 006071 & $2259 \pm 26 \mathrm{AP}$ & $-5,3$ & $1488-1741$ & Valva \\
\hline (3) GUA-010 Conchal & BETA 355645 & $2170 \pm 30 \mathrm{AP}$ & 1,5 & 1363-1627 & Valva \\
\hline \multirow{2}{*}{ (3) GUA-010 Terraza } & BETA 34404 & $5020 \pm 90 \mathrm{AP}$ & $(-)$ & $5583-5918$ & Carbón \\
\hline & BETA 355644 & $5370 \pm 30 \mathrm{AP}$ & $-24,6$ & 5994-6210 & Carbón \\
\hline (3) Isla Solitaria & D-AMS 006072 & $607 \pm 22 \mathrm{AP}$ & $-23,2$ & $529-629$ & Carbón \\
\hline (4) Gran Guaiteca 1 & BETA 355643 & $1120 \pm 30 \mathrm{AP}$ & 2,2 & $440-614$ & Valva \\
\hline (4) Puquitín & UGAMS 8293 & $1050 \pm 30 \mathrm{AP}$ & $-10,1$ & $492-629$ & Colágeno \\
\hline (4) Repollal Cavernas & UGAMS 8295 & $730 \pm 25 \mathrm{AP}$ & $-11,7$ & $141-388$ & Colágeno \\
\hline (5) Repollal Bajo 2 & BETA 355646 & $1960 \pm 30 \mathrm{AP}$ & 1,7 & $1183-1387$ & Valva \\
\hline (6) Isla Sin Nombre & UGAMS 8290 & $870 \pm 30 \mathrm{AP}$ & $-10,4$ & $309-489$ & Colágeno \\
\hline \multirow{2}{*}{ (7) Isla Yalac 1} & UGAMS 26281 & $710 \pm 25 \mathrm{AP}$ & $-12,2$ & 102-335 & Colágeno \\
\hline & UGAMS 26282 & $780 \pm 25 \mathrm{AP}$ & $-11,9$ & $260-436$ & Colágeno \\
\hline \multirow{2}{*}{ (8) Osario Melimoyu } & UGAMS 26280 & $920 \pm 25 \mathrm{AP}$ & $-12,2$ & $363-516$ & Colágeno \\
\hline & UGAMS 26279 & $1010 \pm 25 \mathrm{AP}$ & $-11,7$ & $458-572$ & Colágeno \\
\hline \multirow{2}{*}{ (9) Seno Gala 1} & BETA 230515 & $1340 \pm 40 \mathrm{AP}$ & $-23,6$ & 1093-1299 & Colágeno \\
\hline & BETA 230493 & $1430 \pm 40 \mathrm{AP}$ & $-25,5$ & $1187-1366$ & Carbón \\
\hline \multirow{5}{*}{ (10) Estero Sur } & UGAMS 10822 & $1960 \pm 25 \mathrm{AP}$ & $-12,4$ & 1300-1497 & Colágeno \\
\hline & UGAMS 10818 & $1980 \pm 25 \mathrm{AP}$ & $-11,5$ & $1315-1513$ & Colágeno \\
\hline & UGAMS 10819 & $2030 \pm 25 \mathrm{AP}$ & $-11,9$ & $1356-1555$ & Colágeno \\
\hline & UGAMS 10820 & $2030 \pm 25 \mathrm{AP}$ & $-11,9$ & 1356-1555 & Colágeno \\
\hline & UGAMS 10821 & $2030 \pm 25$ AP & $-11,8$ & 1356-1555 & Colágeno \\
\hline (11) Isla Harris & UGAMS 8289 & $650 \pm 25 \mathrm{AP}$ & $-12,5$ & $1-278$ & Colágeno \\
\hline (12) Canalad 1 & UGAMS 8288 & $740 \pm 30 \mathrm{AP}$ & $-12,0$ & $146-406$ & Colágeno \\
\hline (13) Isla Level 1 & UGAMS 21284 & $120 \pm 25 \mathrm{AP}$ & $-25,0$ & $1-253$ & Carbón \\
\hline \multirow{2}{*}{ (14) Isla Izaza 1} & UGAMS 21286ch & $110 \pm 20 \mathrm{AP}$ & $-25,0$ & Fuera de rango & Carbón \\
\hline & UGAMS $21286\left(^{*}\right)$ & $490 \pm 25 \mathrm{AP}$ & $-0,7$ & Fuera de rango & Valva \\
\hline \multirow{2}{*}{ (14) Isla Izaza 2} & UGAMS 21285ch & $70 \pm 20 \mathrm{AP}$ & $-25,5$ & Fuera de rango & Carbón \\
\hline & UGAMS $21285\left(^{*}\right)$ & $570 \pm 25 \mathrm{AP}$ & 0,5 & Fuera de rango & Valva \\
\hline \multirow{2}{*}{ (15) Isla Ipún 1} & UGAMS 10450 & $870 \pm 25 \mathrm{AP}$ & $-12,8$ & $312-487$ & Colágeno \\
\hline & UGAMS 10451 & $1590 \pm 25 \mathrm{AP}$ & $-9,3$ & $928-1134$ & Colágeno \\
\hline \multirow{5}{*}{ (16) Isla Benjamín 1} & UGAMS 26289 & moderno & $-9,9$ & Fuera de rango & Colágeno \\
\hline & UGAMS 8286 & $700 \pm 30 \mathrm{AP}$ & $-10,6$ & $76-323$ & Colágeno \\
\hline & UGAMS 8287 & $770 \pm 30 \mathrm{AP}$ & $-10,5$ & $241-447$ & Colágeno \\
\hline & UGAMS 26287 & $1590 \pm 25 \mathrm{AP}$ & $-22,7$ & 1371-1518 & Colágeno \\
\hline & UGAMS 26288 & $1820 \pm 25 \mathrm{AP}$ & $-16,7$ & 1607-1748 & Colágeno \\
\hline
\end{tabular}

Tabla 1. Continúa en página siguiente. 


\begin{tabular}{|c|c|c|c|c|c|}
\hline \multirow{3}{*}{ (16) Isla Benjamín 2} & UGAMS 21287ch & $2200 \pm 25 \mathrm{AP}$ & $-26,8$ & $2082-2306$ & Carbón \\
\hline & UGAMS 21288 & $2400 \pm 25 \mathrm{AP}$ & $-25,8$ & $2327-2486$ & Carbón \\
\hline & UGAMS $21287\left(^{*}\right)$ & $2580 \pm 25 \mathrm{AP}$ & $-0,3$ & $1864-2112$ & Valva \\
\hline (16) Isla Benjamín 3 & UGAMS $21289\left(^{*}\right)$ & $2610 \pm 25 \mathrm{AP}$ & 1,0 & $1887-2141$ & Valva \\
\hline (17) Isla Benjamín 4 & UGAMS 21292 & $820 \pm 25 \mathrm{AP}$ & $-10,9$ & $285-453$ & Colágeno \\
\hline \multirow{6}{*}{ (17) Isla Benjamín 5} & UGAMS $21290\left(^{*}\right)$ & $340 \pm 25 \mathrm{AP}$ & $-29,9$ & $302-450$ & Corteza \\
\hline & UGAMS 21451 & $770 \pm 25 \mathrm{AP}$ & $-11,0$ & $248-439$ & Colágeno \\
\hline & UGAMS 21453 & $790 \pm 25 \mathrm{AP}$ & $-11,4$ & $268-438$ & Colágeno \\
\hline & UGAMS $21454\left(^{*}\right)$ & $830 \pm 25 \mathrm{AP}$ & $-11,1$ & $290-459$ & Colágeno \\
\hline & UGAMS 21452 & $850 \pm 25 \mathrm{AP}$ & $-10,9$ & $301-473$ & Colágeno \\
\hline & UGAMS 21291 & $890 \pm 25 \mathrm{AP}$ & $-11,1$ & $323-497$ & Colágeno \\
\hline \multirow{4}{*}{ (18) Isla Elena 1} & UGAMS 8121 & $1750 \pm 30 \mathrm{AP}$ & $-11,2$ & $1087-1283$ & Colágeno \\
\hline & UGAMS 8122 & $1820 \pm 30 \mathrm{AP}$ & $-10,4$ & 1174-1343 & Colágeno \\
\hline & UGAMS 8119 & $1880 \pm 25 \mathrm{AP}$ & $-10,6$ & $1245-1398$ & Colágeno \\
\hline & UGAMS 8120 & $1880 \pm 25 \mathrm{AP}$ & $-10,6$ & $1245-1398$ & Colágeno \\
\hline \multirow{2}{*}{ (19) Isla Victoria 2} & UGAMS 10452 & $1820 \pm 25 \mathrm{AP}$ & $-10,6$ & $1177-133$ & Colágeno \\
\hline & UGAMS 10453 & $2330 \pm 25 \mathrm{AP}$ & $-11,2$ & 1706-1913 & Colágeno \\
\hline \multirow{2}{*}{ (20) Canal Cuche 1} & UGAMS 7749ch & $1960 \pm 25 \mathrm{AP}$ & $-25,4$ & 1818-1927 & Carbón \\
\hline & UGAMS 7749 & $2420 \pm 25 \mathrm{AP}$ & 1,1 & $1675-1922$ & Valva \\
\hline (21) Isla Caniglia & UGAMS 8118 & Moderno & 0,9 & Fuera de rango & Valva \\
\hline (22) Canal Darwin 1 & UGAMS 8116 & $520 \pm 25$ AP & 0,7 & Fuera de rango & Valva \\
\hline (22) Canal Darwin 2 & UGAMS 7750 & $3360 \pm 25 \mathrm{AP}$ & $-0,2$ & $2783-3059$ & Valva \\
\hline \multirow{3}{*}{ (23) Nahuelquín 1} & UGAMS 04949 & $430 \pm 25 \mathrm{AP}$ & -21 & $1-256$ & Colágeno \\
\hline & UGAMS 13127 & $1030 \pm 25 \mathrm{AP}$ & $-11,8$ & $481-619$ & Colágeno \\
\hline & UGAMS 04950 & $1820 \pm 25 \mathrm{AP}$ & $-1,2$ & $1048-1264$ & Valva \\
\hline (24) Isla Acuao 1 & UGAMS 8117 & $1630 \pm 25 \mathrm{AP}$ & $-10,8$ & $963-1173$ & Colágeno \\
\hline \multirow{9}{*}{ (25) Posa Las Conchillas } & UGAMS 7752ch & $1320 \pm 25 \mathrm{AP}$ & -26 & $1172-1277$ & Carbón \\
\hline & UGAMS 7753ch & $1450 \pm 25 \mathrm{AP}$ & $-25,6$ & 1281-1352 & Carbón \\
\hline & UGAMS 7751 & $1580 \pm 25 \mathrm{AP}$ & 1,3 & $780-1015$ & Valva \\
\hline & UGAMS 7752 & $1670 \pm 25 \mathrm{AP}$ & 1,4 & $902-1124$ & Valva \\
\hline & UGAMS 7753 & $1760 \pm 25 \mathrm{AP}$ & 0 & $971-1216$ & Valva \\
\hline & UGAMS 7755 & $1800 \pm 25 \mathrm{AP}$ & 0,4 & 1023-1256 & Valva \\
\hline & UGAMS 7754 & $1810 \pm 25 \mathrm{AP}$ & 1,4 & $1038-1261$ & Valva \\
\hline & UGAMS 7755ch & $3110 \pm 25 \mathrm{AP}$ & $-25,5$ & $3206-3364$ & Carbón \\
\hline & UGAMS 7754ch & $3180 \pm 25 \mathrm{AP}$ & $-24,8$ & $3251-3446$ & Carbón \\
\hline \multirow{2}{*}{ (26) Corrientes del Yates 5} & UGAMS 13128 & $1420 \pm 20 \mathrm{AP}$ & $-11,4$ & $758-929$ & Colágeno \\
\hline & BETA $350305\left(^{*}\right)$ & $1920 \pm 30 \mathrm{AP}$ & $-24,1$ & $1734-1890$ & Carbón \\
\hline (27) Isla Prieto 1 & BETA $350307\left(^{*}\right)$ & $1710 \pm 30 \mathrm{AP}$ & $-13,2$ & $928-1163$ & Valva \\
\hline (28) Península Taitao 5 & BETA $350308\left(^{*}\right)$ & $2850 \pm 30 \mathrm{AP}$ & 0,4 & $2153-2453$ & Valva \\
\hline (29) Isla Goñi 1 & BETA $350306\left(^{*}\right)$ & $4070 \pm 30 \mathrm{AP}$ & $-23,7$ & $4417-4583$ & Carbón \\
\hline
\end{tabular}

Nota: Confeccionada a partir de los datos de Porter (1993), Reyes et al. $(2007,2011,2015,2016,2018,2019) .\left(^{*}\right)$ Edades originales de este artículo. Números de la primera columna indican ubicación en Figura 1.

Tabla 1. Edades radiocarbónicas del archipiélago de los Chonos.

factores ambientales y geomorfológicos (Reyes et al. 2018), como también al tipo de los emplazamientos seleccionados y al uso humano del borde costero (Reyes et al. 2015; Reyes 2017). Las evidencias son limitadas en cuanto a frecuencia y se extenderían al menos desde el Holoceno medio (Porter 1993; Reyes et al. 2016), lo que permite caracterizar la zona como de ocupación marginal en términos geográficos (sensu Borrero 2004). Una ventaja de este carácter marginal es que abre la posibilidad 
de medir las ocupaciones humanas como pulsos en los cuales los momentos con presencia humana se alternan con períodos de vacío. Esta condición, a su vez, ofrece una alta sensibilidad de la señal arqueológica (Méndez et al. 2016), lo que propone un escenario ideal para evaluar la ocupación y desocupación de este territorio archipelágico. Creemos que la ocupación del archipiélago no se expresó uniformemente, sino que debió ser discontinua y en ningún momento intensa.

\section{ÁREA DE ESTUDIO Y MUESTRA}

El archipiélago de los Chonos está compuesto por más de 150 islas que forman una densa red de canales y fiordos de costas abruptas y estrechas (Borgel 1970; Camus 2001). Se extiende entre el sur del archipiélago de Chiloé (4350’ S) y la península de Taitao $\left(46^{\circ} 50^{\prime} \mathrm{S}\right)$. Cubre un área de $\sim 54.000$ $\mathrm{km}^{2}$, con $\sim 360 \mathrm{~km}$ de extensión norte-sur y $150 \mathrm{~km}$ este-oeste. Para contar con una muestra geográfica representativa de la ocupación del archipiélago y su cronología se prospectaron a la fecha un total de $567 \mathrm{~km}$ de borde costero efectivo, desde el borde continental a canales interiores e islas off-shore (isla Guafo), aplicando similar intensidad de trabajo arqueológico (registros, sondeos, excavaciones) y considerando sectores proporcionales con igual intensidad de cobertura (Reyes 2017).

De los 78 sitios arqueológicos identificados se fecharon 42 (material carbonizado orgánico $\mathrm{N}=18$, valvas de moluscos $N=23$, restos óseos humanos $\mathrm{N}=37$, restos óseos de fauna $\mathrm{N}=7$ y una muestra de corteza de árbol trabajada, Tabla 1). Estas muestras proceden de todos los tipos de sitios descritos para el área (Reyes et al. 2015), con excepción de los corrales de pesca y las sendas históricas, que no pudieron ser fechados.

Se consideró principalmente el fechado del inicio de las bases de los depósitos $(N=24)$ de manera de obtener edades mínimas para geoformas costeras ocupadas (Reyes et al. 2018). Edades en distintos puntos de las secuencias estratigráficas permitieron comprender las tasas de sedimentación, así como los eventos de ocupación y abandono, y las tasas de descarte. Así, de los 24 sitios con cronología de la base, 18 incluyen dos o más fechas. De igual manera, se fecharon todos los contextos fúnebres individuales y colectivos (osarios), tanto superficiales como estratigráficos. En el último caso se intentó datar el $100 \%$ de los individuos identificados sobre la base de la cuantificación de NMI (Buikstra y Ubelaker 1994). Ello permitió comprender que, en algunos contextos, los individuos fueron depositados contemporáneamente o en un corto lapso, mientras que en otros hubo reutilización de estos con fines fúnebres con siglos de distancia. Así, los eventos individuales generaron conjuntos colectivos diacrónicos (Reyes et al. 2015; Reyes 2017).

En este trabajo también incorporamos la información cronológica poscontacto occidental (1553 DC) (De Goicueta 1879 [1557-1558]). Corresponde a sitios cholgueros o campamentos formados por las actividades artesanales e industriales extractivas de maderas, mariscos y pieles, con manifiesta intensidad entre el siglo XIX y hasta mediados del siglo XX en las costas de este archipiélago (Curry 1991; Martinic 2005). Así, de los 26 sitios arqueológicos cholgueros identificados, fechamos siete en los inicios de la formación de los depósitos, de manera de obtener edades mínimas para el origen de las ocupaciones.

\section{MÉTODO}

Los fechados radiocarbónicos -que corresponden a análisis con técnica AMS (excepto BETA 34404 convencional) (Porter 1993)- fueron realizados en Beta Analityc Inc., Direct AMS y Center for Applied Isotope Studies de la University of Georgia. Los protocolos de procesamiento de las muestras de cada uno de los laboratorios se encuentran disponibles en línea. ${ }^{1,2}$ Para este trabajo se obtuvieron once edades, mientras que las restantes se encuentran previamente publicadas (Tabla 1).

Todas las fechas de radiocarbono $(\mathrm{N}=86)$ se corrigieron calibrando a $2 \sigma$ con Calib 7.0.4 (Stuiver et al. 2013) y se expresan en años antes del presente (cal. AP). Para las muestras de materiales pertenecientes a cadenas tróficas terrestres se aplicó la curva ShCal13 (Hogg et al. 2013). Un efecto reservorio local de $221 \pm 40$ años se aplicó para las muestras de cadenas tróficas marinas de acuerdo con los valores disponibles más cercanos (Ingram y Southon 1996). Para las muestras de valvas y vertebrados marinos se utilizó este valor al $100 \%$ con la curva Mixed Marine SoHem (Reimer et al. 2013). En el caso de los humanos -dado que el conjunto se caracteriza por una dieta eminentemente marina con una media de $\delta^{15} \mathrm{~N}$ de $17,2 \%{ }^{-}$, se consideró un valor de $80 \%$ con la curva Mixed Marine SoHem (Barberena 2008; Reyes et al. 2019). Por 
su parte, para el único individuo poscontacto con una dieta mixta $\left(\delta^{15} \mathrm{~N}\right.$ de $11.3 \%$ ) se consideró un valor de $50 \%$.

Las series de fechados radiocarbónicos expresados en sumas de probabilidades se usaron para comparar la distribución cronológica. Este es un método difundido de análisis estadístico que permite entender conjuntos de datos cronológicos y sus magnitudes a lo largo del eje temporal y, como tal, proponer hipótesis respecto de las relaciones entre el cambio del ambiente y los procesos culturales (Gamble et al. 2004; Williams 2012). Los resultados presentan la variabilidad en cuanto a la distribución espacial y los materiales fechados. El archipiélago de los Chonos fue dividido operacionalmente en sector norte $\left(43^{\circ} 50^{\prime}-45^{\circ} \mathrm{S}\right)$ y sur $\left(45^{\circ}-46^{\circ} 50^{\prime} \mathrm{S}\right)$ para discutirse en una escala regional y luego en una macroescala, comparando la ocupación humana con áreas aledañas.

\section{RESULTADOS}

De las 86 edades que componen la muestra una sobrerrepresentación)-, las curvas de distribución cronológica no mostraron ninguna variación destacable.

En primer término, el registro ${ }^{14} \mathrm{C}$ del archipiélago de los Chonos muestra una distribución discontinua que comienza en el Holoceno medio con fechas mucho más frecuentes hacia los últimos 2000 años (Figura 2). La distribución temporal del archipiélago norte se compone de 57 edades y corresponde al $66,3 \%$ de la muestra total. Dado que el archipiélago sur incluye el restante $33,7 \%$, es esperable que las tendencias temporales globales del archipiélago estén principalmente influenciadas por la mayor muestra del primero. Sin embargo, si bien es cierto que se observa una edad de ocupación inicial más temprana para el archipiélago norte, $~ 6200$ cal. AP, es destacable que en el quinto milenio AP aparezcan ocupaciones en ambos sectores. Después de estos pulsos puntuales de ocupación, se observan vacíos del orden de milenios en ambas zonas. La presencia humana retorna hacia $\sim 3400$ años cal. AP en el archipiélago sur y hacia 2500 años cal. AP en el archipiélago norte. estudiada, ocho fueron excluidas, ya sea por ser consideradas modernas o fuera del rango de calibración de ShCal13, especialmente al aplicarse el efecto reservorio. Solo se combinaron dos fechados estadísticamente indiferenciables procedentes de un mismo individuo (Benjamín 5, individuo 2: UGAMS 21454 y UGAMS 21291) (Ward y Wilson 1978). No se discute la cronología promediando edades estadísticamente indiferenciables procedentes de un mismo sitio (e.g., osarios) pues, cuando se aplicó esta combinación -en los pocos casos que ameritaba (de manera de evitar

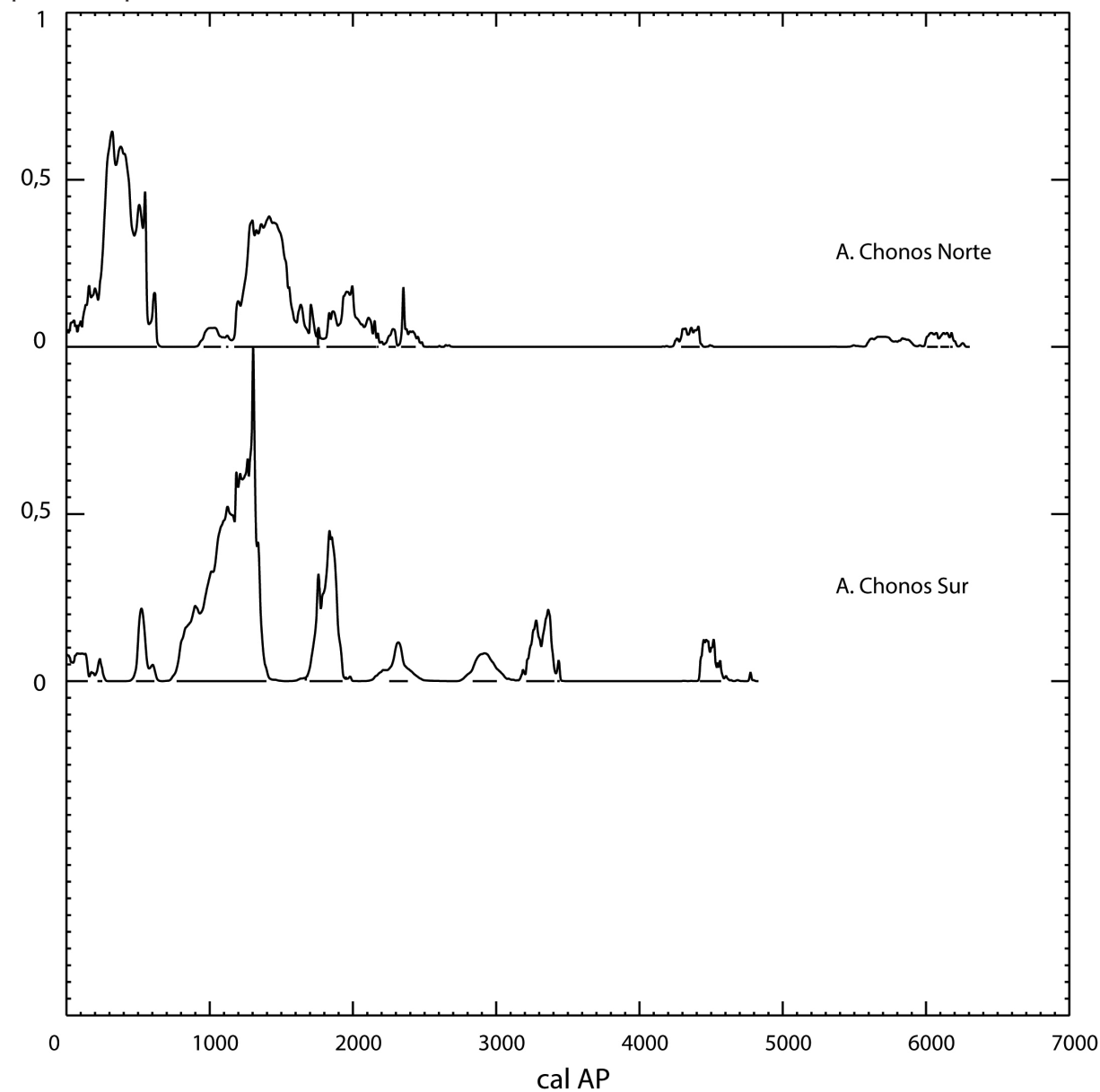

Figura 2. Distribución cronológica de la ocupación humana del archipiélago de los Chonos dividida en sectores norte y sur. 
Para comprender posibles sesgos en la conformación de la muestra, comparamos su distribución temporal de acuerdo con los materiales fechados con el propósito de discutir su conservación diferencial (Figura 3). Mientras que los restos de material vegetal terrestre (95\% carbón) se registran para todo el período en discusión, los restos de valvas de moluscos marinos y el colágeno de hueso y diente (humanos, fauna terrestre y estuarina) tienen una distribución mucho más restringida. Las valvas muestran degradación significativa en los depósitos conchíferos a cielo abierto debido a la alta precipitación y a la formación de suelos muy ácidos (podzol) que caracterizan a la región (Holdgate 1961; Reyes et al. 2016). Este puede ser uno de los factores que inciden en que no registremos depósitos conchíferos con edades superiores a 3100 años cal. AP, pese a haber estudiado unidades del paisaje que potencialmente los tuvieron (Reyes et al. 2018). El material óseo, especialmente los huesos humanos, se encuentra bien conservado dentro de las matrices con valvas, y particularmente dentro de las cuevas secas. Sin embargo, dado que las edades se restringen a los últimos dos milenios, no podemos descartar factores tafonómicos de destrucción que hayan operado en estos materiales. El patrón funerario tipo osario (depositación superficial y subsuperficial) contribuye a la alta representación de fechas tardías en huesos (Reyes 2017).

La distribución general de la cronología del archipiélago de los Chonos muestra un periodo en que la señal es discontinua entre $\sim 6200$ y 2500 años cal. AP (Figura 4). Un segundo bloque corresponde a un periodo en el cual se concentra fuertemente la señal cronológica entre los 2500 años cal. AP y su merma hacia el último siglo. En este segundo bloque observamos una marcada interrupción de la señal ${ }^{14} \mathrm{C}$ hacia $\sim 700$ años cal. AP. Esta predata en $\sim 200$ años a la fecha propuesta para el contacto histórico de acuerdo con los documentos escritos (De Goicueta 1879 [1557-1558]). Aunque con diferencias en magnitud y extensión, esta discontinuidad se observa también en ambos sectores del archipiélago (principalmente en el norte) y en cada uno de los tipos de materiales datados, por lo que interpretamos que corresponde a una tendencia propia de la ocupación humana.

\section{DISCUSIÓN}

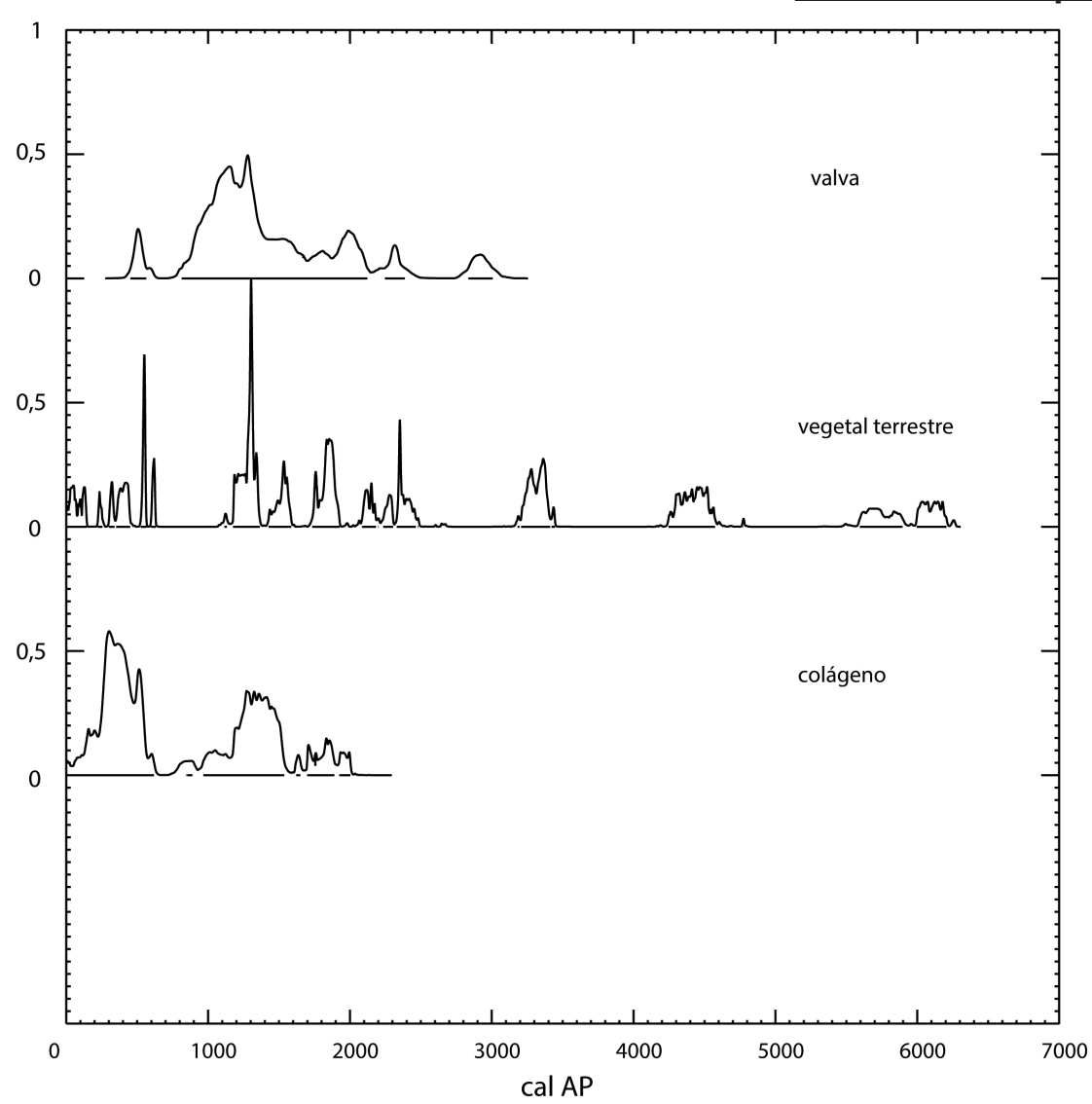

Figura 3. Distribución cronológica de la ocupación humana del archipiélago de los Chonos dividida por materiales fechados. ciones discontinuas entre 6200 y 2500 años cal. AP

La antigüedad máxima de ocupación humana registrada en el archipiélago de los Chonos se remonta al Holoceno medio. En el 010 Terraza presenta dos edades estadísticamente diferentes $(2 \sigma)$, que representan eventos cronológicos independientes iniciados hacia los 6210-5990 años cal. AP (Porter 1993; Reyes et al. 2016). Este sitio está ubicado al borde de una terraza marina emergida a 5,5 m de altitud y a $50 \mathrm{~m}$ de la actual línea de costa (Figura 5). El depósito alterna unidades estratigráficas de arcillas y limos arenosos con la presencia de horizontes de suelo muy orgánicos (podzol altamente ácido; sector norte, el sitio GUA- 


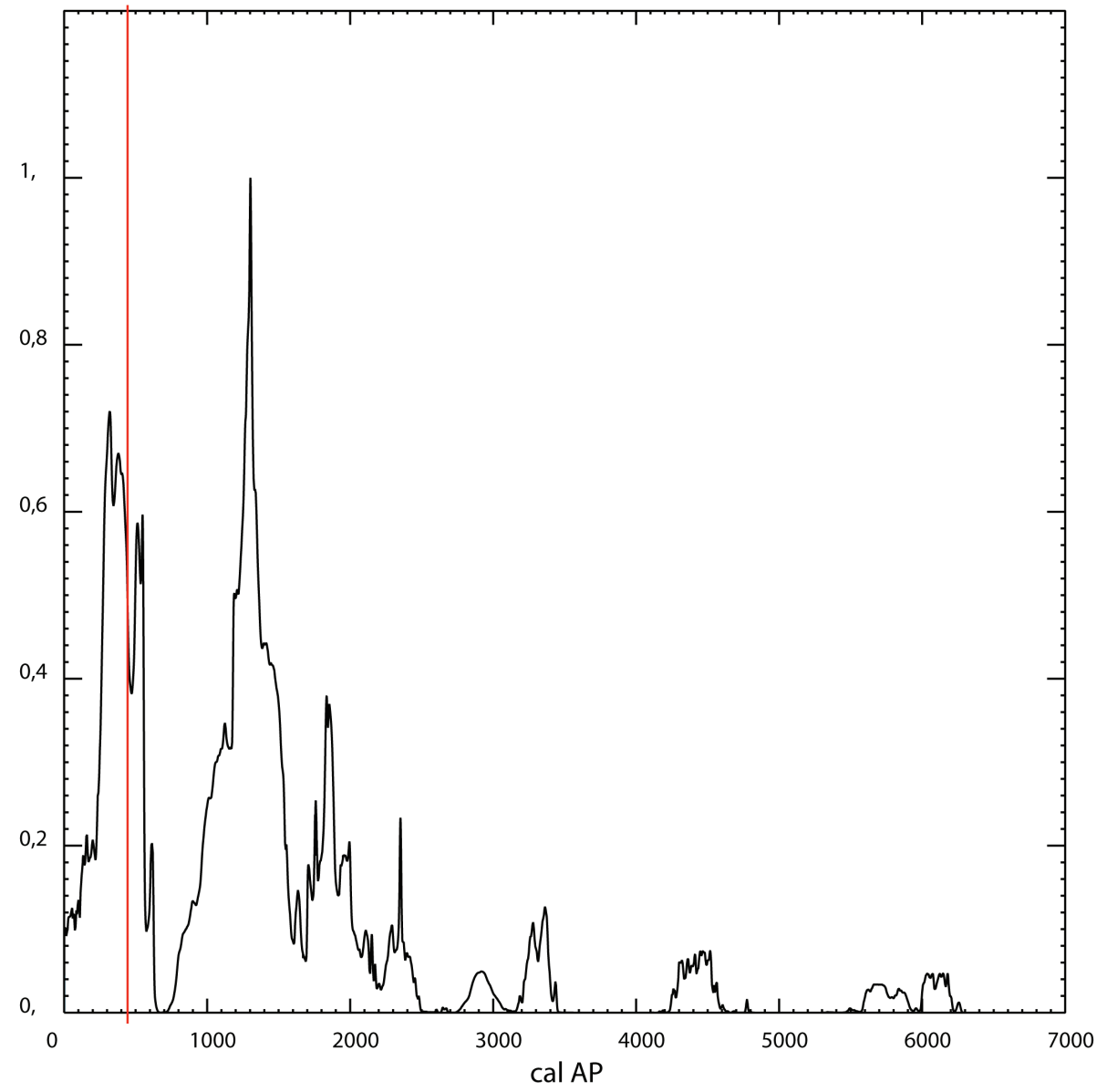

Figura 4. Distribución cronológica de la ocupación humana del archipiélago de los Chonos. La línea roja indica fecha para el contacto local con europeos en península de Taitao de acuerdo con los registros escritos (1553 DC).

pH entre 2,9 y 4,5). Se recuperó exclusivamente material lítico, cuya superficie se observa afectada químicamente. La conservación de estos suelos no permitió la preservación de material faunístico, como tampoco de valvas de molusco. Los materiales líticos indican principalmente actividades de mantención y descarte de cabezales lanceolados de tamaño mediano (8 a $12 \mathrm{~cm}$ ) y sección espesa $(\sim 1 \mathrm{~cm})$. Entre las rocas usadas se registró obsidiana riolítica procedente del volcán Chaitén, distante a $150 \mathrm{~km}$ al noreste en el borde continental (Reyes et al. 2016; Méndez et al. 2018).

Después de un periodo de 1000 años sin señal radiocarbónica, detectamos ocupaciones tanto en el sector norte del archipiélago -en el sitio Gran Guaiteca 2 Terraza hacia 4240-4430 cal. AP (Reyes et al. 2016)-, como en el sector sur en el sitio Isla Goñi 1 -hacia 4420-4530 cal. AP (Reyes 2017)-. Las edades muestran una distribución latitudinal de ocupaciones que incluye de forma sincrónica a los extremos del archipiélago (Reyes 2017). Estos sitios presentan características similares entre sí y también respecto de los eventos de ocupación detectados en GUA-010 Terraza. Los sitios de esta antigüedad no presentan ocupaciones posteriores superpuestas. Todos ellos fueron localizados en emplazamientos que sufrieron profundos cambios geomorfológicos en relación con la posición de las líneas costeras (Reyes et al. 2016, 2018). Es así como registramos solevantamiento y alejamiento de la línea litoral actual en el caso de los sitios del sector norte (GUA-010 Terraza y Gran Guaiteca 2 Terraza), y hundimiento y destrucción en el sector sur del archipiélago (Isla Goñi 1, Figura 5) (Reyes 2017). Las áreas de los sitios intervenidas representan sectores de actividades específicas; sin embargo, no descartamos otras actividades que puedan estar igualmente encubiertas y/o destruidas. Ninguno de los sitios registra evidencia arqueofaunística, y en ambos casos, los materiales líticos fueron recuperados dentro del podzol. El conjunto artefactual de Isla Goñi 1 permitió inferir actividades como la pesca, trabajo sobre madera y caza (pesas de red, hachas líticas y cabezales líticos lanceolados). Observamos también evidencias de la mantención de instrumentos y el uso de obsidiana riolítica de la fuente del volcán Chaitén. La ubicación de estos sitios indica un rango mayor de dispersión desde la fuente de origen (>350 km) hacia el quinto milenio AP. Estas evidencias señalan un uso pleno de la tecnología de navegación, la que habría facilitado el transporte de volúmenes mayores de obsidiana, como lo sugieren las evidencias de lascas con corteza en el sitio Isla Goñi 1 (Reyes 2017).

Posteriormente a una discontinuidad en la señal radiocarbónica de $~ 750$ años se manifiestan las primeras ocupaciones que incluyeron depósitos de 


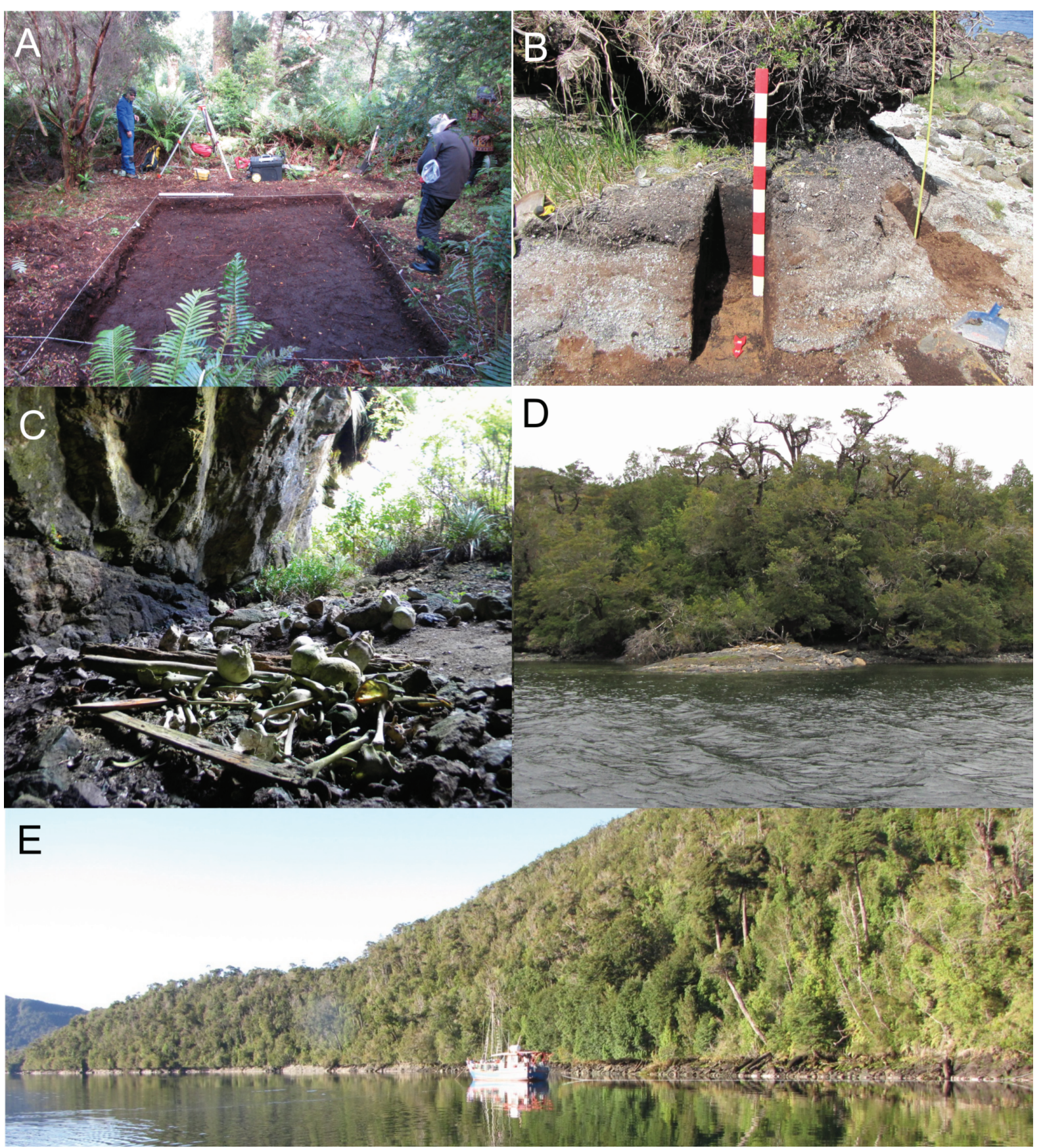

Figura 5. Sitios del archipiélago de los Chonos. A. GUA-010 Terraza; B. Isla Goñi 1; C. Isla Yalac 1; D. Península de Taitao 5; E. Paisaje característico del archipiélago de los Chonos donde se aprecia vegetación tupida en ladera abrupta que colinda con el mar.

valvas de moluscos hacia los 3500 años cal. AP. Destacan sitios como Posa las Conchillas y Canal Darwin 2, en el sector sur del archipiélago. A diferencia de los milenios anteriores, se observa un uso reiterado de las localidades a partir de la superposición de eventos de ocupación, incluso con depósitos de hasta $8 \mathrm{~m}$ de espesor (Reyes et al. 2015). Las valvas se constituyen como una de las partículas sedimentarias antropogénicas más relevantes en la conformación de estos depósitos (Stein 1992). En estos concheros se registran fogones, espacios de descarte de basura, talla lítica e inhumaciones, que representan diversas actividades propias de los campamentos formados por estos grupos canoeros.

\section{Periodo de ocupaciones semicontinuas: 2500 años cal. AP al presente}

A partir de los 2500 años cal. AP se inicia una señal radiocarbónica semicontinua en el archipiélago de los Chonos. Muestra su punto máximo hacia los $~ 1300$ años cal. AP y luego una merma 
notoria hacia los 700 años cal. AP. Ambos sectores del archipiélago se comportan de forma común. Un segundo punto máximo relevante -que solo se observó en el sector norte- ocurre hacia los 300 años cal. AP, el que coincide plenamente con el contacto europeo. Durante este bloque temporal registramos un aumento de los eventos de ocupación relacionados con una mayor cantidad y diversidad de los sitios (avistaderos, concheros a cielo abierto y bajo reparo, osarios). Una importante cantidad de dispersiones superficiales de material lítico en el intermareal posiblemente se hayan producido durante este intervalo, en vista de su asociación espacial con contextos fechados (Reyes et al. 2007, 2016). Una situación análoga podría sugerirse para los corrales de pesca registrados (Reyes et al. 2015).

El patrón de emplazamiento de las localidades ocupadas fue eminentemente de borde costero, con reocupaciones en los mismos lugares, que dieron origen a grandes montículos concheros. Al igual que en el periodo anterior, los cambios geomorfológicos permanentes de esta zona fueron constatados en todo el archipiélago, donde el hundimiento tectónico provocó la erosión de los perfiles expuestos a las mareas y la consecuente dispersión del material lítico y bioantropológico en el área intermareal (Reyes et al. 2018). Además, se ocuparon otros lugares -como reparos rocosos-, cuya locación no excede los 50 metros de distancia de la costa actual. Estos sitios, aun estando disponibles, no fueron ocupados en momentos anteriores. En islas off-shore como Guafo, no registramos evidencia de ocupaciones prehistóricas (Reyes 2017), lo que nos señala hasta ahora que la exploración y ocupación del archipiélago no fue total. Por otro lado, reparos rocosos, como Alero Low e Isla Benjamín 1, fueron empleados inicialmente para actividades domésticas y, hacia los últimos momentos de su ocupación, se registró un cambio de función del sitio hacia fines fúnebres. Los conjuntos artefactuales asociados a los sitios de este periodo muestran una prevalencia del uso de recursos líticos locales procurados en el entorno y combinados con obsidiana del volcán Chaitén. Esto reafirma una continuidad del uso de este recurso clave a lo largo de toda la secuencia de ocupación del archipiélago. Por su parte, los conjuntos faunísticos de los concheros muestran una prevalencia de la explotación de recursos malacológicos complementados con aves marinas, peces, mamíferos ribereños $y$, en mucha menor medida, mamíferos marinos y terrestres. Esta selectividad se encuentra en sintonía con los valores de $\delta^{13} \mathrm{C}$ y $\delta^{15} \mathrm{~N}$ obtenidos de los restos humanos recuperados del archipiélago (Reyes et al. 2019).

El aspecto más notable dentro de este bloque temporal es la gran profusión de restos humanos, cuenta que asciende a 203 individuos en el archipiélago (Reyes 2017). La evidencia bioantropológica más temprana en un osario bajo reparo rocoso corresponde al individuo 2 de Isla Victoria 2 (1710-1910 años cal. AP). Por su parte, la edad más temprana de una inhumación en conchero corresponde a un individuo recuperado del intermareal de Isla Acuao 1 (960-1170 años cal. AP). Los osarios muestran tanto contextos donde múltiples individuos fueron depositados de manera sincrónica (edades estadísticamente indiferenciables que conforman un único evento ocupacional: Estero Sur e Isla Benjamín 1), como osarios formados por la reiteración de eventos distanciados en el tiempo (Alero Low, Isla Ipún 1, Osario Melimoyu, Isla Yalac 1, Isla Victoria 2) (Figura 5). También se detectaron dos casos de osarios donde se combinaron eventos sincrónicos y diacrónicos (Isla Benjamín 5 e Isla Elena 1). Junto con los osarios, restos humanos fueron recuperados de la intervención de nueve concheros. Los restos frecuentemente se observan en perfiles erosionados o desperdigados en el intermareal. Ahora, la mayor visibilidad de los osarios en cuevas sesga la muestra en favor de este tipo de conjuntos.

Respecto de la distribución cronológica de este periodo, Ilaman especialmente la atención los comportamientos que ocurrieron en momentos previos y posteriores al contacto europeo. Estas tendencias deben ser consideradas preliminares en tanto los ajustes de efecto reservorio local no existan. Los grupos humanos que habitaban el archipiélago sufrieron un proceso de desmantelamiento cultural acelerado en menos de 200 años (Fitz-Roy 1839; Urbina 1988; Urbina 2016). Observamos una notoria baja en la curva previo al contacto europeo ( 700 años cal. AP) y posteriormente un repunte en tiempos poscontacto (peak: 300 años cal. AP). Las razones para la marcada merma aún son desconocidas; sin embargo, variables tafonómicas (e.g., destrucción de sitios por cambios geomorfológicos) y el diseño de búsqueda no explican completamente esta dinámica en el registro (Reyes et al. 2015, 2018). Se ha postulado un descenso en la demografía como resultado de la expansión de las poblaciones agricultoras del norte, que provocaron el inicio de una crisis adaptativa anterior al contacto local con los europeos (1553 DC) (Aspillaga et al. 
2006). Por su parte, no obstante la ocupación europea involucró traslados forzados (e.g., misiones, encomiendas) fuera del archipiélago, el aumento de eventos cronológicos poscontacto pudo deberse a que algunos grupos canoeros se adecuaron a las nuevas condiciones de relaciones interétnicas y comerciales que estimularon las visitas a esta área (Urbina et al. 2019). Esta mayor presencia pudo incidir en el incremento de señal radiocarbónica poscontacto.

En forma posterior al siglo XIX, los cambios en las relaciones comerciales involucraron nuevos tipos de ocupaciones del archipiélago. Los cholgueros como manifestación arqueológica más visible corresponden a grandes acumulaciones, principalmente de Mytilus, formadas por las ocupaciones estacionales, recurrentes y con fines comerciales de pescadores, peleteros y hacheros. A partir de estos registramos materialmente la intensificación en la obtención de recursos marinos específicos y a escala comercial (Martinic 2005). Estas ocupaciones, aun cuando fueron fechadas, no se representan en la curva radiocarbónica puesto que resultaron modernas o fuera del límite de calibración. Sin embargo, la profusión y magnitud de este tipo de sitios muestran una intensidad de uso sin precedentes en esta área insular.

\section{Comparaciones regionales}

Lo observado en el archipiélago de los Chonos durante el Holoceno medio contrasta con las evidencias reportadas para el extremo septentrional de los canales de Patagonia $\left(\sim 41-43^{\circ} \mathrm{S}\right)$, donde los sitios arqueológicos ubicados en el seno de Reloncaví (i.e., Piedra Azul, Centro Acuicultura Metri) y en la isla de Chiloé (i.e., Puente Quilo, Chepu, Yaldad 2) evidencian cronologías levemente más antiguas, con reiteración en el uso de los espacios, que incluyen ocupaciones más tardías con la presencia de cerámica (Rivas et al. 1999; Gaete et al. 2004; Ocampo y Rivas 2004; Legoupil 2005; Rivas y Ocampo 2006; Flores et al. 2010; Flores y Correa 2011). La situación particular del archipiélago de los Chonos sugiere un periodo exploratorio inicial con una ocupación más efímera y discontinua, de acuerdo con la cual los emplazamientos más antiguos no volvieron a ser frecuentados (Reyes 2017).

El registro arqueológico durante el Holoceno tardío en el seno de Reloncaví y Chiloé, en tanto, se caracteriza por una mayor diversidad en cuanto a los depósitos y tipos de registro (Flores et al. 2010). Entre las evidencias se incluyen grandes concheros monticulares multicomponentes, depósitos efímeros con materiales cerámicos, pinturas rupestres y grabados en cuevas, sitios funerarios, además de corrales de pesca (Munita et al. 2005, 2011; Aspillaga et al. 2006; Labarca et al. 2016). Aunque en el archipiélago de los Chonos aumentan la diversidad y densidad del registro arqueológico, este no refleja toda la variabilidad de ocupaciones de más al norte, sino solamente algunas de ellas (Reyes et al. 2015). Sin embargo, en coherencia con los conjuntos de fechados ${ }^{14} \mathrm{C}$ cuantificados para regiones aledañas y para Patagonia en general, se registra una tendencia hacia el incremento de la señal cronológica humana, lo que en conjunto ha sido postulado como un aumento demográfico (Pérez et al. 2016).

A nivel de macroescala espacial, al contemplar toda el área de los canales occidentales de Patagonia y del archipiélago fueguino, observamos que la mayor antigüedad de ocupación registrada hasta ahora en el archipiélago de los Chonos es más reciente que las detectadas en las áreas litorales más septentrionales y meridionales (Orquera et al. 2011). No obstante, la sincronía del surgimiento de la navegación durante el sexto milenio y el modo de vida canoero en varias regiones de la Patagonia occidental y Tierra del Fuego indica que este proceso ocurrió en un corto periodo de tiempo y tuvo una amplia dispersión geográfica (Orquera y Piana 2006; Orquera et al. 2011). La información recabada aún no permite sugerir direccionalidad en la propagación de este fenómeno. Sin embargo, la discontinuidad en el proceso puede ser entendida de modo preliminar como una característica propia de lo ocurrido en este espacio, a diferencia de zonas donde el registro arqueológico aparece representado de forma más reiterada una vez iniciadas la colonización y ocupación humanas.

\section{CONCLUSIONES}

La recopilación de fechados radiocarbónicos producidos por la investigación continua de más de una década en el archipiélago de los Chonos permite evaluar los pulsos de las ocupaciones humanas que lo habitaron durante el Holoceno. Las tendencias generales observadas tuvieron un similar comportamiento al analizar comparativamente la muestra entre los sectores norte y sur; como entre distintos materiales fechados (i.e., colágeno de 
hueso, material carbonizado vegetal y valvas). Ello sugiere que no hubo un sesgo influenciado por los conjuntos numéricamente más relevantes, como el de los restos producto del registro de osarios. Esto apunta a que la distribución es representativa de las tendencias temporales del archipiélago.

La distribución temporal de ocupaciones en el área de estudio entre los 6200 y 2500 años cal. AP se caracteriza por la discontinuidad general del registro arqueológico. El registro generado por los grupos cazadores recolectores marinos muestra una intensidad de ocupación significativamente baja, con grandes hiatos de desocupación a escala de milenios. Desde los 2500 años cal. AP en adelante, los sitios se distribuyen de manera más uniforme, y cubren latitudinalmente desde la costa continental hasta el borde del Pacífico, exceptuando islas offshore como Guafo. Una mayor variabilidad, que incluye campamentos concheros, incrementó la visibilidad arqueológica asociada a la redundancia en el uso de algunas locaciones. Después de un máximo en la distribución temporal hacia los 1300 años cal. AP, la señal cronológica cae dramáticamente hasta sus niveles más bajos. De manera preliminar, inferimos una discontinuidad en el uso del archipiélago entre los 800 y 650 años cal. AP. El contacto europeo local hacia 1553 DC no coincide con esta caída dramática, sino que después de los primeros encuentros documentados, el área muestra el segundo aumento en los datos de radiocarbono. Aunque consideramos que los datos son preliminares, las reconfiguraciones en el uso del espacio producto de la conquista europea pueden explicarse por movimientos poblacionales y cambios en las relaciones productivas.

En el contexto del desarrollo cultural de los grupos canoeros en Patagonia septentrional, el archipiélago de los Chonos representa una extensa zona insular cuyas visitas y ocupaciones fueron muy poco frecuentes prácticamente a lo largo de toda su secuencia. Esta representa una zona altamente sensible a las influencias de los cambios externos visibles a partir del registro de radiocarbono. Es por ello que constituye un caso de estudio para comprender las dinámicas poblacionales en ambientes insulares marginalmente ocupados.

\section{Agradecimientos}

Financiamiento FONDECYT 1170726 y 1130151 , FONDECYT Regional R17A10002. Agradecemos a
Amalia Nuevo Delaunay por la ayuda con la confección de la Figura 5 y a los tres evaluadores de este trabajo por sus oportunas observaciones.

\section{REFERENCIAS CITADAS}

Aspillaga, E., M. Castro., M. Rodríguez y C. Ocampo 2006 Paleopatología y estilo de vida: El ejemplo de los Chonos. Magallania 34 (1): 77-85.

Barberena, R.

2008 Arqueología y biogeografía humana en Patagonia Meridional. Sociedad Argentina de Antropología, Buenos Aires.

Borgel, R.

1970 Geomorfología de las regiones australes de Chile. Revista Geográfica de Chile Terra Australis 20: 135-140.

Borrero, L.

2004 The Archaeozoology of Andean "Dead Ends" in Patagonia: Living near the Continental Ice Cap. Colonisation, Migration, and Marginal Areas. En $A$ Zooarchaeological Approach, editado por M. Mondini, S. Muñoz y S. Wickler, pp. 55-61. Oxbow Books, Oxford.

Buikstra, J. y D. Ubelaker 1994 Standards for Data Collection from Human Skeletal Remains. Proceedings of a Seminar at the Field Museum of Natural History. Arkansas Archeological Report Research Series, Fayetteville.

Camus, $\mathrm{P}$.

2001 Biogeografía marina de Chile continental. Revista Chilena de Historia Natural 74: 587-617.

Curry, P.

1991 Distribución de sitios e implicaciones para la movilidad de los canoeros en el canal Messier. Anales del Instituto de la Patagonia 20: 145-154.

De Goicueta, $M$.

1879 [1557-1558] Viaje del capitán Juan Ladrillero al descubrimiento del Estrecho de Magallanes. Anuario Hidrográfico de la Marina de Chile. Año V. Imprenta Nacional, Santiago.

De la Fuente, C., C. Ávila, J. Galimany, M. Carpenter, J. Homburger, A. Blanco, P. Contreras, D. Cruz, O. Reyes, M. San Román, A. Moreno, P. Campos, C. Eng, S. Huntsman, E. Burchard, C. Bustamante, E. Willerslev, E. Llop, R. Verdugo y M. Moraga 2018 Genomic insights into the origin and diversification of late maritime hunter-gatherers from the Chilean Patagonia. PNAS 115 (17): E4006-E4012. 
Fitz-Roy, R.

1839 Voyages of the Adventure and Beagle. Volume II. Proceedings of The Second Expedition, 1831-1836. Henry Colburn, Londres.

Flores, C. y I. Correa

2011 Explotación de ambientes costeros, intensificación de recursos y transformaciones culturales en la transición Holoceno medio a Holoceno tardío en la costa de los canales patagónico septentrionales, Chile. Actas XVII Congreso Nacional de Arqueología Argentina. Tomo I: 321-326

Flores, C., B. Broitman y P. Rivas

2010 Changes in the subsistence strategy of prehistoric intertidal gathering: the pre-ceramic and ceramic coastal hunter-gatherers of Reloncaví Sound, Chile. En Comparative Perspectives on the Archaeology of Coastal South America, pp. 63-76. Center for Comparative Archaeology, University of Pittsburgh, Pontificia Universidad Católica del Perú, Ministerio de Cultura del Ecuador.

Gaete, N., X. Navarro, F. Constantinescu, R. Mera, D. Selles, M. Solari, L. Vargas, D. Oliva y L. Durán 2004 Una mirada al modo de vida canoero del mar interior desde Piedra Azul. Chungara Revista de Antropología. Volumen especial I: 333-346.

Gamble, C., W. Davies, P. Pettitt y M. Richards 2004 Climate change and evolving human diversity in Europe during the last glacial. Philosophical Transactions of the Royal Society B. 359: 243-254.

Hogg, A., Q. Hua, P. Blackwell, M. Niu, C. Buck, T. Guilderson, T. Heaton, J. Palmer, P. Reimer, R. Reimer, C. Turney y S. Zimmerman

2013 SHCal13 Southern Hemisphere calibration, 0-50,000 years cal BP. Radiocarbon 55 (4): 1889-1903.

Holdgate, $M$.

1961 Vegetation and Soils in the South Chilean Islands. Journal of Ecology 49 (3): 559-580.

Ingram, B. L. y J. Southon

1996 Reservoir ages in eastern Pacific coastal and estuarine waters. Radiocarbon 38 (3): 573-582.

Kuzminsky, S., O. Reyes, B. Arriaza, C. Méndez, V. Standen, M. San Román, I Muñoz y A. Durán

2018 Investigating Cranial Morphological Variation of Early Human Skeletal Remains from Chile: A 3D Geometric Morphometric Approach. American Journal of Physical Anthropology 165 (2): 223-237.

Labarca, R., F. Mena, A. Prieto, T. Dupradou y E. Silva 2016 Investigaciones arqueológicas en torno a los primeros registros de Arte Rupestre en Morro Vilcún.
Boletín Museo Chileno de Arte Precolombino 21 (2): 65-80.

Legoupil, D.

2005 Recolectores de mariscos tempranos en el sureste de la isla de Chiloé. Magallania 33 (1): 51-61.

Martinic, M

2005 De la Trapananda al Aysén. Pehuén, Santiago.

Méndez, C. y O. Reyes

2015 Archaeology near the southern ice-end. Current advances in human interdisciplinary research in central western Patagonia. SAA Archaeological Record 15 (3): 21-26.

Méndez, C., M. E. de Porras, A. Maldonado, O. Reyes, A. Nuevo y J. García

2016 Human effects in Holocene fire dynamics of Central Western Patagonia ( 44 $\mathrm{S}$, Chile). Frontiers in Ecology and Evolution 4: 100.

Méndez, C., C. Stern, A. Nuevo, O. Reyes, F. Gutiérrez y F. Mena

2018 Spatial and temporal distributions of exotic and local obsidians in Central Western Patagonia, southernmost South America. Quaternary International 468: 155-168.

Munita, D., R. Álvarez y C. Ocampo

2005 Corrales de piedra, pesca pasiva en la costa interior de Chiloé. Boletín de la Sociedad Chilena de Arqueología 37: 61-74.

Munita, D., R. Mera, I. Arregui y M. Manneschi 2011 Funebria de grupos canoeros durante el holoceno tardío en la región de los lagos. El conchal de Yaco Alto-1, Calbuco, Chile. Revista Cazadores Recolectores del Cono Sur. Revista de Arqueología 6: 17-41.

Ocampo, C. y P. Rivas

2004 Poblamiento temprano de los extremos geográficos de los canales patagónicos: Chiloé e isla Navarino 1. Chungara Revista de Antropología. Volumen especial I: 317-331.

Orquera, L., D. Legoupil y E. Piana 2011 Littoral adaptation at the southern end of South America. Quaternary International 239: 61-69.

Orquera, L. y E. Piana

2006 El poblamiento inicial del área litoral sudamericana sudoccidental. Magallania 34 (2): 21-36.

Pérez, I., P. González y V. Bernal 2016 Past population dynamics in Northwest Patagonia: An estimation using molecular and radiocarbon data. Journal of Archaeological Science 65: 154-160. 
Porter, C.

1993 GUA-010, un sitio costero erosionado en una zona sísmica activa. Actas del XII Congreso Nacional de Arqueología Chilena. Boletín del Museo Regional de la Araucanía 4: 81-88.

Reimer, P., E. Bard, A. Bayliss, J. Beck, P. Blackwell, C. Bronk Ramsey, C. Buck, H. Cheng, R. Edwards, M. Friedrich, P. Grootes, T. Guilderson, H. Haflidason, I. Hajdas, C. Hatté, T. Heaton, A. Hogg, K. Hughen, K. Kaiser, B. Kromer, S. Manning, M. Niu, R. Reimer, D. Richards, E. Scott, J. Southon, C. Turney y J. van der Plicht 2013 IntCal13 and MARINE13 radiocarbon age calibration curves 0-50000 years cal BP. Radiocarbon 55 (4): 1869-1887.

Reyes, O.

2017 El poblamiento del Archipiélago de los Chonos $\left(43^{\circ}-47^{\circ} \mathrm{S}\right)$. Patagonia Occidental, Chile. Tesis Doctoral inédita. Facultad de Filosofía y Letras, Universidad de Buenos Aires, Buenos Aires.

Reyes, O., C. Méndez, M. San Román, P. Cárdenas, H. Velásquez, V. Trejo, F. Morello y C. Stern

2007 Seno Gala 1; Nuevos resultados en la arqueología de los Canales Septentrionales (44 S, XI Región de Aisén, Chile). Magallania 35 (2): 105-119.

Reyes, O., M. San Román y M. Moraga

2011 Archipiélago de los Chonos: Nuevos registros arqueológicos y bioantropológicos en los canales septentrionales. Isla Traiguén, XI Región de Aisén. Magallania 39 (2): 295-303.

Reyes, O. M. Moraga, C. Méndez, y A. Cherkinsky 2015 Maritime hunter-gatherers in the Chonos

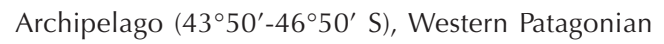
Channels. Journal of Island and Coastal Archaeology 10 (2): 207-231.

Reyes, O. M. San Román y F. Morello 2016 Search for maritime hunter-gatherer archaeological record in the shifting shorelines of the South Pacific Coast (Chonos and Guaitecas Archipelago, Chile). En Marine Ventures: Archaeological Perspectives on Human-Sea Relations. Equinox Publishing, United Kingdom, editado por H. B. Bjerck, H. M. Breivik, S. E. Fretheim, E. Piana, B. Skar, A. M. Tivoli y A. F. Zangrando, pp. 141-155. Equinox, Sheffield, Reino Unido.

Reyes, O., C. Méndez, M. San Román y J. Francois 2018 Earthquakes and coastal archaeology: Assessing shoreline shifts on the southernmost Pacific coast

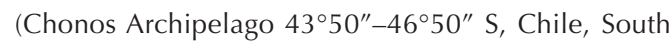
America). Quaternary International 463: 161-175
Reyes, O., A. Tessonne, M. San Román y C. Méndez 2019 Dieta e isótopos estables de cazadores recolectores marinos en los canales occidentales de Patagonia, Chile. Latin American Antiquity. En prensa.

Rick, J. W.

1987 Dates as Data: An examination of the Peruvian Preceramic radiocarbon record. American Antiquity 52: 55-73.

Rivas, P. y C. Ocampo 2006 La adaptación humana al bosque en la isla de Chiloé. Estrategias adaptativas en el litoral septentrional de los canales patagónicos. Actas del XVII Congreso Nacional de Arqueología Chilena. Tomo 2: 1449-1460.

Rivas, P., C. Ocampo y E. Aspillaga

1999 Poblamiento Temprano de los Canales Patagónicos: El Núcleo Ecotonal Septentrional. Anales del Instituto de la Patagonia 27: 221-230.

Stein, J.

1992 Sediment analysis of the British Camp shell midden. En Deciphering a Shell Midden, editado por J. Stein, pp. 135-162. Academic Press, San Diego.

Stuiver, M., P. Reimer y R. Reimer

2013 CALIB 7.0.4 www program and documentation. http://calib.qub.ac.uk/calib/

Urbina, R.

1988 Los Chonos en Chiloé: Itinerario y Aculturación. Revista Chiloé 9: 29-42.

Urbina, $X$.

2016 Traslados de indígenas de la Patagonia occidental insular a Chiloé en los siglos XVI, XVII y XVIII. En América en diásporas: esclavitudes y migraciones forzadas (siglos XVI-XIX), pp. 381-411. Instituto de Historia, Pontificia Universidad Católica de Chile/RiL editores, Santiago.

Urbina, X., O. Reyes y C. Belmar 2019 Canoeros en Chiloé: de facilitadores de las navegaciones españolas en los archipiélagos de los Chonos y de Guayaneco, a productores y comerciantes, 1567-1792. MS.

Ward, G. y S. Wilson

1978 Procedures for comparing and combining radiocarbon age determinations: a critique.

Archaeometry 20 (1): 19-31.

Williams, A.

2012 The use of summed radiocarbon probability distributions in archaeology: A review of methods. Journal of Archaeological Science 39: 578-589. 
Cronología de la ocupación humana en los canales septentrionales de Patagonia occidental... Intersecciones en Antropología 20 (2), agosto-diciembre. 2019. ISSN-e 1850-373X

\section{NOTAS}

1.- https://www.radiocarbon.com/espanol/acelerador-masaespectrometria.htm

2.- https://cais.uga.edu/service/radiocarbon-dating-by-ams/ 
210 | O. Reyes et al. - Intersecciones en Antropología 20 (2), agosto-diciembre. 2019. ISSN-e 1850-373X 